\title{
Herramientas que mejoran el diagnóstico. Inteligencia artificial
}

\author{
Miguel Á. Herrera-Servin* \\ Departamento de Endoscopia, Hospital General de México Dr. Eduardo Liceaga, Ciudad de México, México
}

\section{Introducción}

La inteligencia artificial (IA) se está abriendo camino en el mundo de la endoscopia, ha pasado ya la fase de experimentación para abrirse a la fase de detección en vivo, e incluso retar a sus maestros. Llego para quedarse, pero ahora debemos preguntarnos ¿Cuál será su lugar en nuestro ejercicio diario? A continuación, se presentan aquellos trabajos donde se demuestra la utilidad de la IA en los diferentes segmentos del tubo digestivo. Al final de todo esto debemos pensar en los retos o dudas de su aplicación que nos quedan en el aire.

\section{IA en endoscopia superior}

En el estudio de Choi, et al. ${ }^{1}$, se realizó un modelo de IA para detección de displasia en imágenes, con el propósito de detectar neoplasia esofágica temprana en videos de casos con esófago de Barrett. Fue un total de 20 videos (de 1 a 6 minutos de duración); 10 de pacientes con displasia y 10 de pacientes sin displasia (todos se realizaron con luz blanca y NBI). Los resultados en el modelo de IA demostraron sensibilidad del $91 \%$ para detectar lesiones y $3.7 \%$ de falsos positivos, sin la necesidad de tener que detener o congelar la imagen. Falta demostrar su utilidad en casos in vivo.

Fukuda, et al. ${ }^{2}$, presentaron otro estudio, aunque con respecto al cáncer de células escamosas de esófago. En este compararon los resultados de la $|A+N B|$ contra endoscopistas expertos (13 endoscopistas certificados), para el diagnóstico en tiempo real de CCE de esófago. Se utilizaron 7,181 imágenes sin magnificación y 7530 con magnificación de 1571 lesiones superficiales de CCE; 564 imágenes sin magnificación y 2744 con magnificación de mucosa esofágica normal. Se realizaron videos de 5 a 9 segundos de 144 pacientes. Los resultados fueron: para la IA sensibilidad, especificidad y precisión para detectar CCE de 91, 51 y $63 \%$, respectivamente. Para los expertos; sensibilidad, especificidad y precisión de 79,72 y $75 \%$. Aunque la sensibilidad de la IA fue mayor, la especificidad estuvo a favor del panel de expertos.

Al caracterizar las lesiones la IA tuvo una sensibilidad, especificidad y precisión del $86 \%, 89$ y $88 \%$ mientras que para los expertos la sensibilidad, especificidad y precisión fueron de 74,76 y $75 \%$, respectivamente; teniendo la IA un mejor desempeño en este punto, siendo esta combinación comparable y significativamente superior a la de los endoscopistas expertos.

\section{IA en colonoscopía (26 a $30 \%$ de lesiones perdidas)}

Entre las áreas hacia las que se ha dirigido más la IA se encuentran la detección y caracterización de lesiones tempranas, siendo un ejemplo de esto el metaanálisis presentado por Ka-Luen Lui $\mathrm{L}$, et al. ${ }^{3}$. Este incluyó 3 estudios prospectivos con 4754 imágenes de 16 colonoscopias, con el objetivo de 
determinar la precisión diagnóstica de la IA en la predicción histológica y detección de pólipos colorrectales. Los resultados fueron: precisión de la IA de 0.96 (IC 95\%: 0.94-0.98) para detectar la presencia de lesiones neoplásicas en términos de AUC, sensibilidad $92.6 \%$ (IC 95\%: 89.0-95.4) y especificidad 89.2\% (IC 95\%: 84.7-92.8). El AUC se vio incrementada en aquellas imágenes donde se utilizó NBI (AUC 0.98; IC 95\%: 0.97-0.99 vs. 0.84; IC 95\%: 0.76-0.92; $p<0.01)$.

Para la caracterización de pólipos diminutos se obtuvo sensibilidad de $94 \%$, especificidad de $91.3 \%$, VPN 0.91 , con desempeño mayor comparado a endoscopista no experto (AUC 0.97; IC 95\%: 0.96-0.98 vs. 0.90; IC 95\%: 0.87-0.93; $p<0.01)$. En la detección de pólipos alcanzó AUC 0.90; (IC 95\%: 0.67-1.00), sensibilidad 95\% (IC 95\%: 91.0-97.0) y especificidad 88.0\% (IC 95\%: 58.0-99.0), demostrando la superioridad de la $|A+N B|$ para la detección y caracterización de pólipos en comparación con endoscopistas no expertos (aunque se necesitan más estudios).

En referencia a otras tecnologías de detección, Neumann, et al. ${ }^{4}$ analizaron la eficacia de la IA uniendo sistema CAD (Deep-learning computer-aided detection) con LCl (Fujifilm) para la detección de pólipos colorrectales. Se entrenó una CNN con imágenes de pólipos vistos bajo cromoendoscopia digital $\mathrm{LCl}$ y para la validación de la red se analizaron videos completos de colonoscopías con detección de 240 pólipos y confirmación histológica de adenomas.

Los resultados fueron: sensibilidad del $100 \%$ sin lesiones perdidas para el sistema CAD-LCl, con rango por cuadro de falsos positivos de $0.001 \%$. 34 de estos pólipos eran serrados y fueron detectados al $100 \%$. Se concluyó que esta combinación podría ser útil para la detección de pólipos (especialmente serrados) en colon.

En otro trabajo (retrospectivo y prospectivo) presentado por Ka-Luen Lui, et al. ${ }^{5}$ se analizó comparó el porcentaje de lesiones pérdidas durante la colonoscopia para la IA y el colonoscopista. Durante el estudio endoscópico (de forma blindada) se revisó el colon, mientras en un monitor aparte un investigador observaba el resultado de la máquina, si está ultima encontraba un pólipo desapercibido para el endoscopista, se le notificaba y se revisaba nuevamente el segmento. Los resultados fueron: detección por la IA de $79 \%$ de adenomas perdidos (principalmente en colon proximal). Obteniendo mayor eficacia al realizar la revisión en conjunto con el endoscopista.

\section{IA en CPRE y USE}

Durante este año, entre los trabajos que se presentaron sobre el uso de IA en USE, con el objetivo de valorar la utilidad de la IA para distinguir entre pancreatitis autoinmune (PAI) de adenocarcinoma ductal pancreático (ADP) ${ }^{6}$, se utilizaron 1.2 millones de imágenes de 576 pacientes (144 PAl, 288 ADP, 72 pancreatitis crónica y 72 páncreas normal) tomadas de videos de USE. Se entreno a una red ResNet50 CNN. 80\% de los casos fueron utilizados para el entrenamiento de la red y $20 \%$ para la prueba.

Los resultados fueron: La IA tiene una sensibilidad y especificidad de $93 \%$ y $90 \%$ respectivamente con área bajo la curva de 0.963 para el diagnóstico de PAl.

El principal predictor de PAI fue el reforzamiento hiperecoico entre la interfase del páncreas con las estructuras adyacentes (53\% PAl contra 0\% de ADP; $p<0.01)$. La mejora post-acústica profunda de ductos/vasos fue altamente predictiva de ADP ( $57 \%$ ADP vs. $0 \%$ PAl; $p<0.01$ ).

Se concluyó que el USE-CNN es útil para diferenciar entre estas patologías. Las características de EUS de PAl ocurren más comúnmente dentro del páncreas o en los planos de tejido entre el parénquima y otras estructuras. Para ADP, las características únicas de EUS con mayor frecuencia involucran sitios peripancreáticos y retroperitoneales.

\section{Financiamiento}

Los autores no recibieron patrocinio para llevar a cabo este artículo.

\section{Conflicto de intereses}

Los autores declaran no tener conflicto de intereses alguno.

\section{Bibliografía}

1. Choi AY, Hashimoto R, Cavaliere KR. High accurancy and effectiveness with deep neural networks and artificial intelligence in detection of early esophageal neoplasia in barrett's esophagus: an external video validation study. DDW. 2020. 2020 Mayo; (Poster Mo1249-2020).

2. Fukuda $H$, Ishihara $R$, Kato $Y$. Comparison of artificial intelligence and expert endoscopist toward real-time assisted diagnosis of esophageal squamous cell carcinoma. DDW2020. 2020 mayo;(poster Sa2057-2020).

3. Ka-Luen Lui T, Guo CG, Keung Leung W. Accuracy of artificial intelligence on histological prediction and detection of colorectal polyps: a systematic review and metaanalysis. DDW. 2020. 2020 Mayo; (Poster Sa1662-2020).

4. Neumann H, Sivanathan V, Fareed Rahman K. Artificial intelligence combined with Ici yields in highest accuracy and detection of colorectal polyps, including sessile serrated lesions. DDW. 2020. 2020 Mayo; (Poster Sa 2042-2020).

5. Ka-Luen Lui T, Hui C, Tsui VW. Artificial intelligence-assisted real-time detection reduces missed lesions during colonoscopy: a retrospective and prospective study. DDW 2020. 2020 Mayo; (Poster Sa1998-2020).

6. Mayra N, Powers P, Chari S. Application of artificial intelligence in differentiating aip from pdac using eus-convolutional neural network image analysis and feature extraction techniques. DDW 2020.2020 Mayo; (Poster 660_2020). 